\title{
OPTICALLY-SWITCHED MICROWAVE FILTER WITH THE USE OF PHOTOVARACTORS IN SELF-BIAS MODE
}

\author{
Zenon R. Szczepaniak*, Bogdan A. Galwas ${ }^{\star}$ and Sergei A. Malyshev ${ }^{\#}$ \\ *\& Institute of Microelectronics and Optoelectronics, Warsaw University of Technology \\ Nowowiejska 15/19, 00-665 Warsaw, Poland \\ Phone/Fax: (+48 22) 8250393 \\ *zenon.szczepaniak@elka.pw.edu.pl, ${ }^{\&}$ B.Galwas@elka.pw.edu.pl \\ \# Institute of Electronics, National Academy of Sciences of Belarus \\ 22 Lagoiski Trakt, 220090 Minsk, Republic of Belarus \\ malyshev@inel.bas-net.by \\ Phone: (375-17) 265-22-13, Fax: (375-17) 265-25-41
}

\begin{abstract}
A new type of semiconductor optoelectronic device, which is called as photovaractor, was used in a self-bias mode of operation to obtain an optically-variable impedance. This approach allowed designing an optically-switched microwave band-pass filter. The use of the photovaractor in the microwave structure was investigated. The measurements of the photovaractor, the idea of the selfbias mode, and the simulations of the filter have been presented.
\end{abstract}

\section{INTRODUCTION}

The fast development in the microwaves and the photonic fields causes the merging of these two branches. The microwave-driven optical devices as well as the optically-controlled microwave circuits are needed. Especially, the optically-controlled microwave signal processing is now under the interest, because of the development in the new radar, and fibre-radio systems. It is worth to point out the approach using the microwave planar structures, for example resonators, made on the semiconductor substrate - Cabon et al (1). Another approach to design the optically-controlled microwave circuits is to use two coupled semiconductor devices, when the first one is photosensitive, and the second one is controlled. The examples of such a solution are the following devices couples: PIN photodiode-varactor diode - by Szczepaniak and Galwas (2), photovoltaic cell-varactor diode, by Nagra et al (3), photovoltaic cell-FET - Sun et al (4). An alternative approach is to design and made the semiconductor device which parameters can be optically changed. The examples of this idea are microwave phototransistors and photovaractors.

\section{PHOTOVARACTOR IN SELF-BIAS MODE}

A new type of special microwave junction device is a photovaractor. Generally, it is for example, a PIN photodiode, which is specially designed, and optimised to obtain very high capacitance changes under the optical illumination. Idea, measurements and modelling of the photovaractor were described in previous papers - Malyshev et al (5). However the device provides the impedance change dependent on the bias voltage and the illuminating power level, the new proposal of the operation is a bias-free configuration. The assumption is that the external bias circuit is not used, and moreover, the device has its pins (anode and cathode) short-circuited by a very large resistor for example $1 \mathrm{M} \Omega$. It means that the photodiode is terminated by almost open. Then the optical illumination causes the generation of a voltage across the device. This mode of the operation can be described as a self-bias mode.

In this setup even relatively small optical illumination causes the significant change of the photovaractor impedance. The change of the capacitance as well as resistance is observed. 
An example of the photovaractor impedance changes under the optical illumination for the frequency band from $300 \mathrm{kHz}$ to $3 \mathrm{GHz}$ is shown in Fig. 1, and at the frequency equal to $900 \mathrm{MHz}$ is shown in Fig. 2.

It can be seen that with the use of the optical power equal to about $0.112 \mathrm{~mW}$ it is possible to obtain two photovaractor impedance states: almost open $(\mathrm{ON})$ and almost $50 \Omega$ match $(\mathrm{OFF})$ - Fig. 2.

This capability allows to design several microwave circuits working with the use of the two-state impedance.

\section{OPTICALLY-SWITCHED MICROWAVE FILTER}

The possibility of the optical control of the photovaractor impedance was considered to design an optically-switched microwave filter. The first design was the microwave band-pass filter at the centre frequency about $900 \mathrm{MHz}$. The filter structure consists of two coupled sections of the microstrip line. The second step of the design was the optimisation of the filter structure with two photovaractors connected to it. The general topology and the idea of the filter (it is not real scale) were shown in Fig. 3. The transmission characteristics of the filter with and without the photovaractors were shown in Fig. 4. The filter is in the ON state when the photovaractors are not illuminated. Then the photovaractors impedance is almost open, and the microwave signal can be transmitted through the filter structure. When the photovaractors are illuminated by the optical power, their impedance approaches to $50 \mathrm{ohms}$ and causes the attenuation of the microwave signal, resulting in decreasing of the transmission. The Q-factor of the resonant filter structures is then very low. It corresponds to the OFF state. The transmission characteristics of the microwave filter with the photovaractors in the ON and OFF states were shown in Fig. 5.

\section{CONCLUSIONS}

However the filter without photodiodes can be designed with minimal losses in the pass-band, the filter with photovaractors has higher losses because the photovaractor impedance is not the ideal open. The OFF state can be obtain with the use of the optical power illuminating the photovaractors equal to $0.112 \mathrm{~mW}$. The resistors loading the photovaractors influence on the speed of reaction of the photovaractor impedance under the optical illumination. The decrease of the resistors values will cause the improvement of the switching speed. However, the lower values of the resistors, the higher optical power required to achieve the same photovaractor impedance changes under the illumination. The optically-variable impedance of the photovaractor can be used in other different microwave applications.

\section{REFERENCES}

1. B. Cabon, A. Vilcot, S. Chouteau, B. Boyer, J. Haidar, M. Bouthinon, and J. Boussey - „Optical control of microwave passive devices integrated on silicon", Proc. $10^{T H}$ MICROCOLL Conference 1999, Budapest Hungary March 21-24, 1999.

2. Z. Szczepaniak and B.Galwas, "Optically-Controlled Microwave Phase Shifter", Proc. EDMO'99, King's College London, UK, 22-23 November 1999, pp.290-294.

3. A.S Nagra, O. Jerphagnon, P. Chavarkar, M. VanBlaricum, and R.A. York, "Indirect optical control of microwave circuits using monolithic optically variable capacitors," IEEE Trans. on MTT, Vol. 47, No.7, July 1999, pp. 1365-1372.

4. C. K. Sun, R. Nguyen, C. T. Chang, and D. J. Albares, "Photovoltaic-FET for optoelectronic

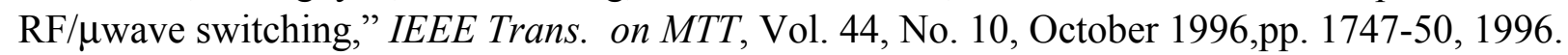

5. S.Malyshev, B.Galwas, V.Andrievski, A.Chizh, L.Dobrzanski, and Z.Szczepaniak, "Analysis of photovaractors for microwave photonic application", Proc. $30^{T H}$ EuMC'2000, France, Paris, 3-5 October 2000, vol.3, pp.210-213. 


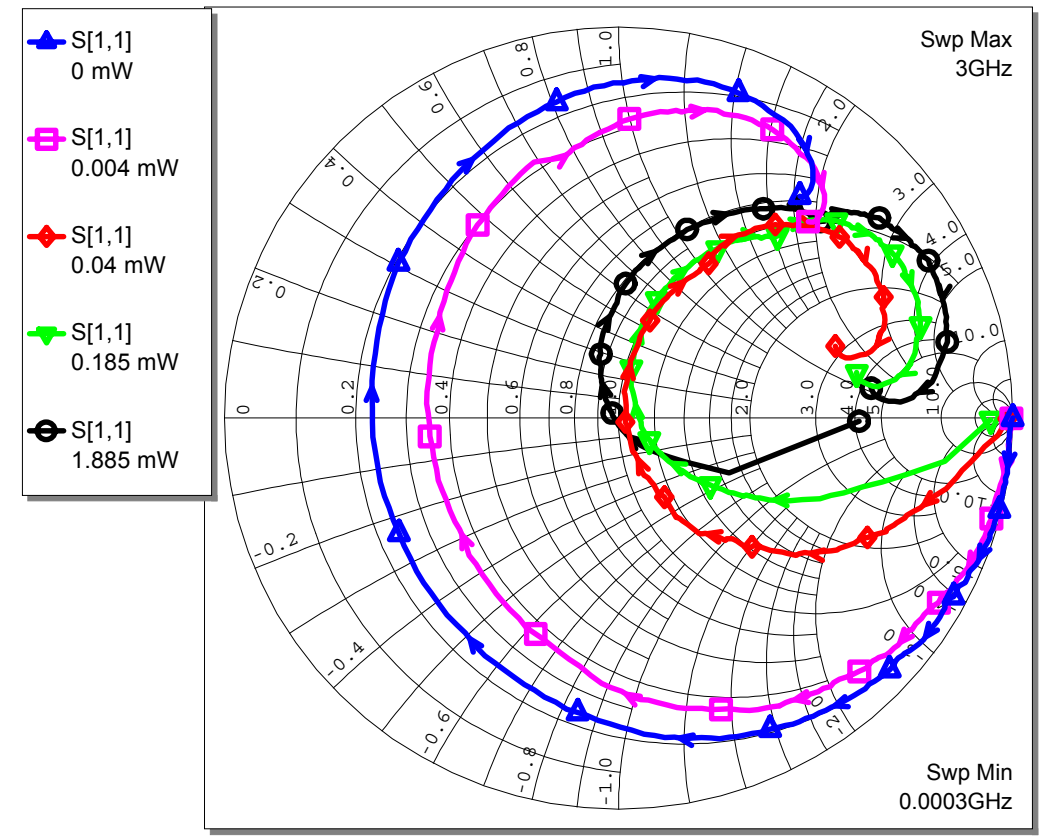

Fig. 1. Photovaractor impedance vs. frequency for different illumination powers in self-bias mode.

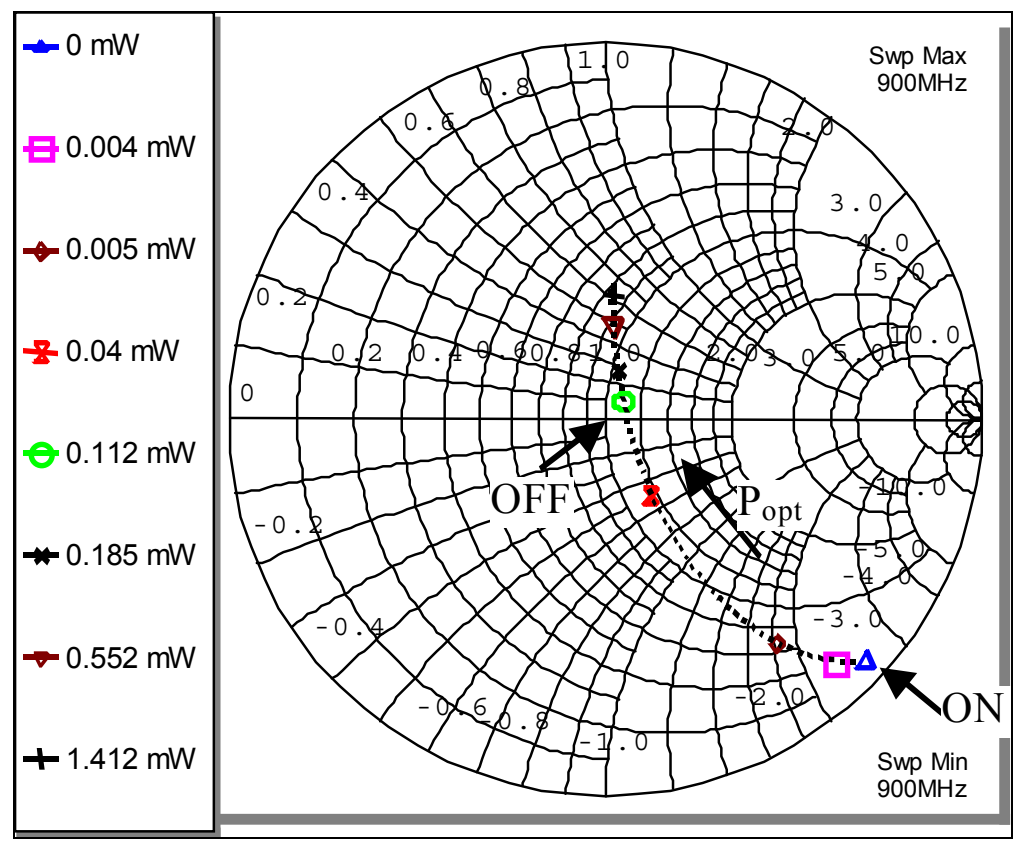

Fig. 2. Photovaractor impedance at $900 \mathrm{MHz}$ for different illumination powers in self-bias mode.

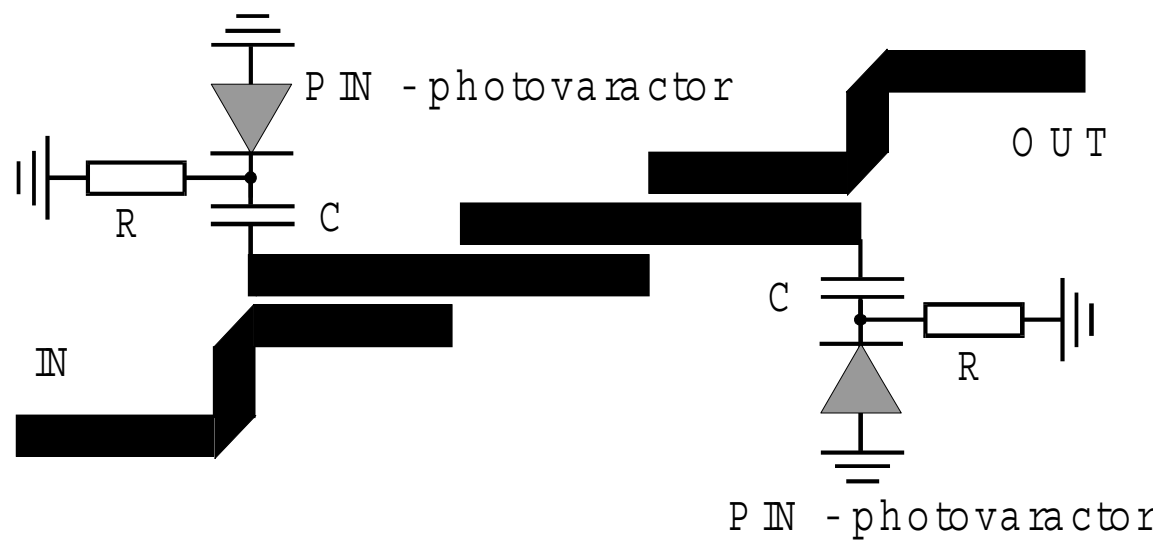

Fig. 3. Proposed idea of optically-controlled microwave filter. 


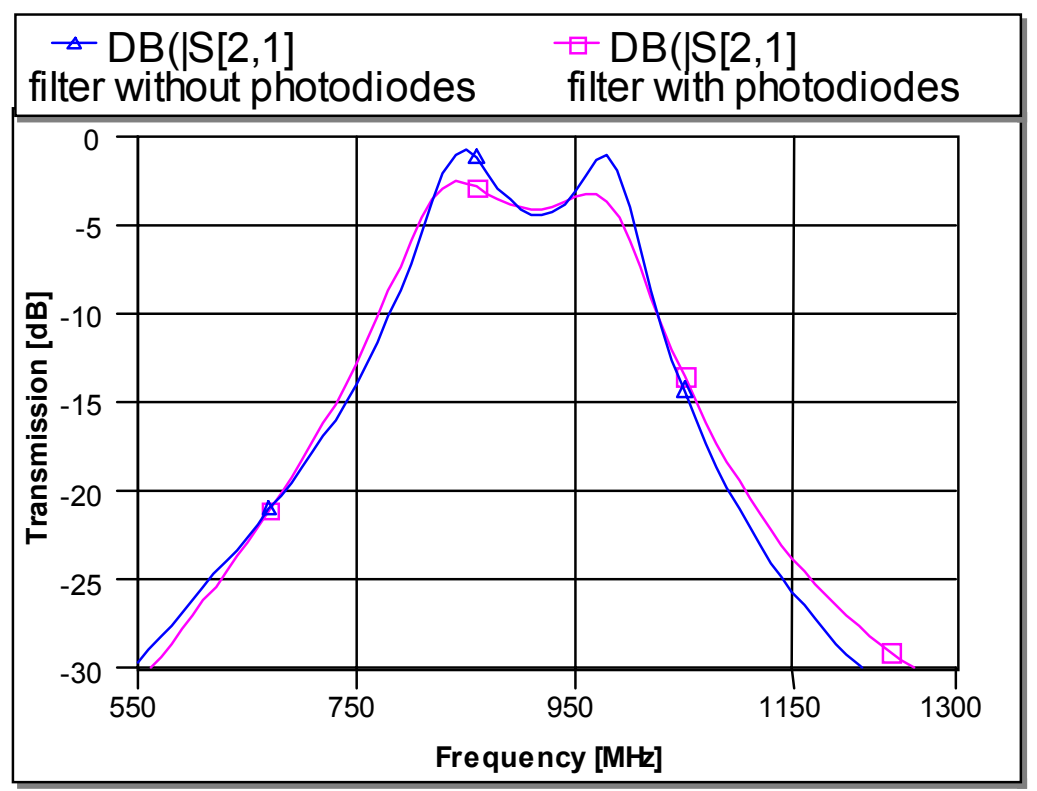

Fig. 4. Transmission characteristics of the filter without photovaractors and optimised filter with photovaractors.

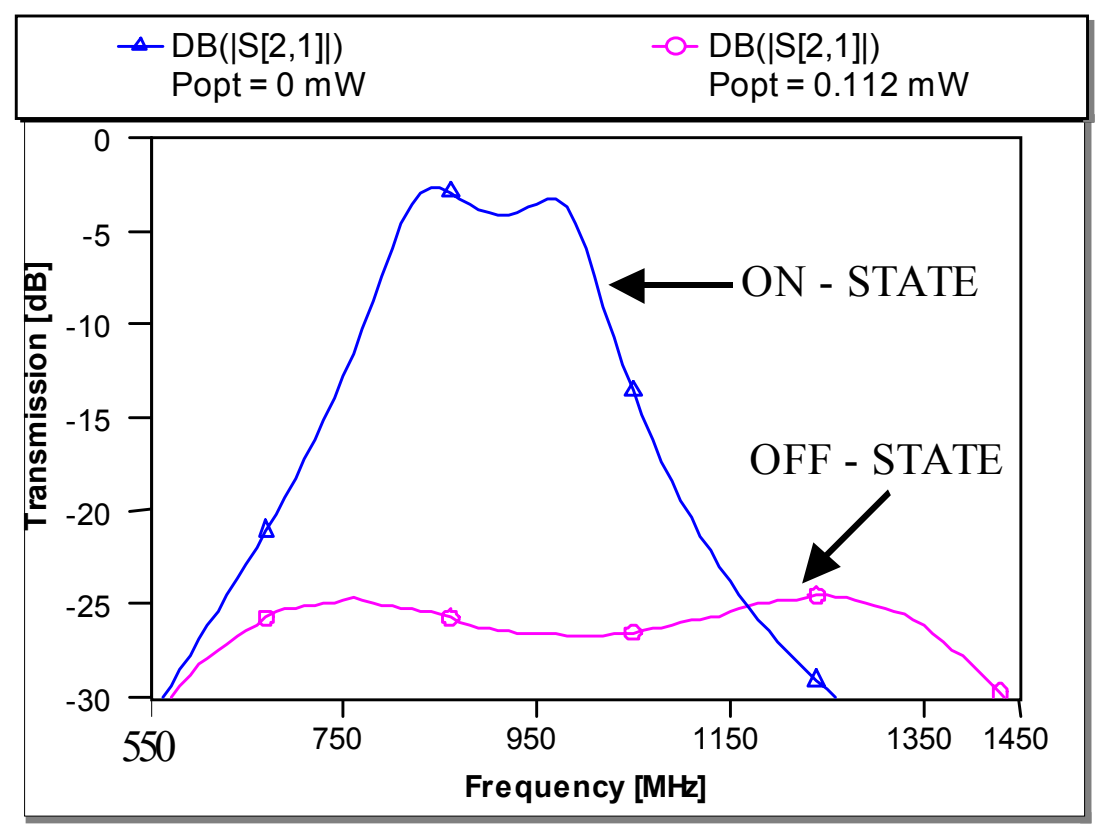

Fig. 5. Transmission characteristics of the microwave filter with the photovaractors in ON (not illuminated) and OFF (illuminated) states. 